\title{
ON THE STABILITY OF THE MIXED TYPE FUNCTIONAL EQUATION IN RANDOM NORMED SPACES VIA FIXED POINT METHOD
}

\author{
Sun SOOK JIN ${ }^{\mathrm{a}}$ AND YANG-Hi LEE ${ }^{\mathrm{b}, *}$
}

\begin{abstract}
In this paper, we prove the stability in random normed spaces via fixed point method for the functional equation

$$
f(x+y+z)-f(x+y)-f(y+z)-f(x+z)+f(x)+f(y)+f(z)=0 .
$$
\end{abstract}

by using a fixed point theorem in the sense of L. Cădariu and V. Radu.

\section{INTRODUCTION}

In 1940, S. M. Ulam [20] raised a question concerning the stability of homomorphisms: Given a group $G_{1}$, a metric group $G_{2}$ with the metric $d(\cdot, \cdot)$, and a positive number $\varepsilon$, does there exist a $\delta>0$ such that if a mapping $f: G_{1} \rightarrow G_{2}$ satisfies the inequality

$$
d(f(x y), f(x) f(y))<\delta
$$

for all $x, y \in G_{1}$ then there exists a homomorphism $F: G_{1} \rightarrow G_{2}$ with

$$
d(f(x), F(x))<\varepsilon
$$

for all $x \in G_{1}$ ? As mentioned above, when this problem has a solution, we say that the homomorphisms from $G_{1}$ to $G_{2}$ are stable. In 1941, D. H. Hyers [5] gave a partial solution of Ulam's problem for the case of approximate additive mappings under the assumption that $G_{1}$ and $G_{2}$ are Banach spaces. Hyers' result was generalized by T. Aoki [1] for additive mappings and Th. M. Rassias [16] for linear mappings by considering the stability problem with unbounded Cauchy differences. The paper

Received by the editors October 25, 2011. Revised February 16, 2012. Accepted Feb. 20, 2012. 2000 Mathematics Subject Classification. Primary 54E40, 39B82, 46S50, 46S40.

Key words and phrases. stability, additive mapping, mixed type functional equation, random normed space.

${ }^{*}$ Corresponding author. 
of Th. M. Rassias has provided a lot of influence in the development of stability problems. The terminology Hyers-Ulam-Rassias stability originated from these historical background. During the last decades, the stability problems of functional equations have been extensively investigated by a number of mathematicians, see [2]-[4], [8]-[12].

Recall, almost all subsequent proofs in this very active area have used Hyers' method, called a direct method. Namely, the function $F$, which is the solution of a functional equation, is explicitly constructed, starting from the given function $f$, by the formulae $F(x)=\lim _{n \rightarrow \infty} \frac{1}{2^{n}} f\left(2^{n} x\right)$ or $F(x)=\lim _{n \rightarrow \infty} 2^{n} f\left(\frac{x}{2^{n}}\right)$. In 2003, V. Radu [15] observed that the existence of the solution $F$ of a functional equation and the estimation of the difference with the given function $f$ can be obtained from the fixed point alternative. In 2008, D. Mihet and V. Radu [14] applied this method to prove the stability theorems of the Cauchy functional equation:

$$
f(x+y)-f(x)-f(y)=0
$$

in random normed spaces. We call solutions of (1.1) additive mappings.

In 2002, S.-M. Jung [7] established the general solution and investigated the stability of the mixed type functional equation:

$$
f(x+y+z)-f(x+y)-f(y+z)-f(x+z)+f(x)+f(y)+f(z)=0
$$

by using the direct method. Now we consider the functional equation:

$$
f(x+y+z)-f(x+y)-f(y+z)-f(x+z)+f(x)+f(y)+f(z)-f(0)=0
$$

which is called the general quadratic functional equation. In this paper, using the fixed point method, we will prove the stability for the functional equation (1.2) and the general quadratic functional equation in random normed spaces. It is easy to see that the mappings $f(x)=a x^{2}+b x$ and $f(x)=a x^{2}+b x+c$ are solutions of the functional equation (1.2) and (1.3), respectively. Every solution of the mixed type functional equation (1.2) and the general quadratic functional equation (1.3) are said to be a quadratic-additive mapping and a general quadratic mapping, respectively.

\section{Preliminaries}

In this section, we state the usual terminology, notations and conventions of the theory of random normed spaces, as in $[18,19]$. Firstly, the space of all probability distribution functions is denoted by 
$\Delta^{+}:=\{F: \mathbb{R} \cup\{-\infty, \infty\} \rightarrow[0,1] \mid F$ is left-continuous and nondecreasing on $\mathbb{R}$, where $F(0)=0$ and $F(+\infty)=1\}$.

And let the subset $D^{+} \subseteq \Delta^{+}$be the set $D^{+}:=\left\{F \in \Delta^{+} \mid l^{-} F(+\infty)=1\right\}$, where $l^{-} f(x)$ denotes the left limit of the function $f$ at the point $x$. The space $\Delta^{+}$is partially ordered by the usual pointwise ordering of functions, that is, $F \leq G$ if and only if $F(t) \leq G(t)$ for all $t \in \mathbb{R}$. The maximal element for $\Delta^{+}$in this order is the distribution function $\varepsilon_{0}: \mathbb{R} \cup\{0\} \rightarrow[0, \infty)$ given by

$$
\varepsilon_{0}(t)= \begin{cases}0 & \text { if } t \leq 0 \\ 1 & \text { if } t>0\end{cases}
$$

Definition 2.1 ([18]). A mapping $\tau:[0,1] \times[0,1] \rightarrow[0,1]$ is called a continuous triangular norm (briefly, a continuous $t$-norm) if $\tau$ satisfies the following conditions:

(a) $\tau$ is commutative and associative;

(b) $\tau$ is continuous;

(c) $\tau(a, 1)=a$ for all $a \in[0,1]$;

(d) $\tau(a, b) \leq \tau(c, d)$ whenever $a \leq c$ and $b \leq d$ for all $a, b, c, d \in[0,1]$.

Typical examples of continuous $t$-norms are $\tau_{P}(a, b)=a b, \tau_{M}(a, b)=\min (a, b)$ and $\tau_{L}(a, b)=\max (a+b-1,0)$.

Definition 2.2. A random normed space (briefly, $R N$-space) is a triple $(X, \Lambda, \tau)$, where $X$ is a vector space, $\tau$ is a continuous $t$-norm, and $\Lambda$ is a mapping from $X$ into $D^{+}$such that the following conditions hold:

(RN1) $\Lambda_{x}(t)=\varepsilon_{0}(t)$ for all $t>0$ if and only if $x=0$,

(RN2) $\Lambda_{\alpha x}(t)=\Lambda_{x}(t /|\alpha|)$ for all $x$ in $X, \alpha \neq 0$ and all $t \geq 0$,

(RN3) $\Lambda_{x+y}(t+s) \geq \tau\left(\Lambda_{x}(t), \Lambda_{y}(s)\right)$ for all $x, y \in X$ and all $t, s \geq 0$.

If $(X,\|\cdot\|)$ is a normed space, we can define a mapping $\Lambda: X \rightarrow D^{+}$by

$$
\Lambda_{x}(t)=\frac{t}{t+\|x\|}
$$

for all $x \in X$ and $t>0$. Then $\left(X, \Lambda, \tau_{M}\right)$ is a random normed space, which is called the induced random normed space.

Definition 2.3. Let $(X, \Lambda, \tau)$ be an $R N$-space.

(i) A sequence $\left\{x_{n}\right\}$ in $X$ is said to be convergent to a point $x \in X$ if, for every $t>0$ and $\varepsilon>0$, there exists a positive integer $N$ such that $\Lambda_{x_{n}-x}(t)>1-\varepsilon$ whenever $n \geq N$. 
(ii) A sequence $\left\{x_{n}\right\}$ in $X$ is called a Cauchy sequence if, for every $t>0$ and $\varepsilon>0$, there exists a positive integer $N$ such that $\Lambda_{x_{n}-x_{m}}(t)>1-\varepsilon$ whenever $n \geq m \geq N$.

(iii) An RN-space $(X, \Lambda, \tau)$ is said to be complete if and only if every Cauchy sequence in $X$ is convergent to a point in $X$.

Theorem $2.4([18])$. If $(X, \Lambda, \tau)$ is an $R N$-space and $\left\{x_{n}\right\}$ is a sequence such that $x_{n} \rightarrow x$, then $\lim _{n \rightarrow \infty} \Lambda_{x_{n}}(t)=\Lambda_{x}(t)$.

\section{Main Results}

We recall the fundamental result in the fixed point theory.

Theorem 3.1 ([13, 17]). Suppose that a complete generalized metric space $(X, d)$, which means that the metric d may assume infinite values, and a strictly contractive mapping $J: X \rightarrow X$ with the Lipschitz constant $0<L<1$ are given. Then, for each given element $x \in X$, either

$$
d\left(J^{n} x, J^{n+1} x\right)=+\infty, \forall n \in \mathbb{N} \cup\{0\},
$$

or there exists a nonnegative integer $k$ such that:

(1) $d\left(J^{n} x, J^{n+1} x\right)<+\infty$ for all $n \geq k$;

(2) the sequence $\left\{J^{n} x\right\}$ is convergent to a fixed point $y^{*}$ of $J$;

(3) $y^{*}$ is the unique fixed point of $J$ in $Y:=\left\{y \in X, d\left(J^{k} x, y\right)<+\infty\right\}$;

(4) $d\left(y, y^{*}\right) \leq(1 /(1-L)) d(y, J y)$ for all $y \in Y$.

Let $X$ and $Y$ be vector spaces. We use the following abbreviations for a given mapping $f: X \rightarrow Y$ by

$$
\begin{aligned}
D f(x, y, z):= & f(x+y+z)-f(x+y)-f(y+z)-f(x+z)+f(x)+f(y)+f(z) \\
D^{\prime} f(x, y, z):= & f(x+y+z)-f(x+y)-f(y+z)-f(x+z)+f(x)+f(y)+f(z) \\
& -f(0)
\end{aligned}
$$

for all $x, y, z \in X$.

Lemma 3.2 ([6]). If $f: X \rightarrow Y$ is a mapping such that $D f(x, y, z)=0$ for all $x, y, z \in X \backslash\{0\}$, then $f$ is a quadratic-additive mapping.

Now we will establish the stability for the functional equations (1.2) in random normed spaces via fixed point method. 
Theorem 3.3. Let $X$ be a linear space, $\left(Z, \Lambda^{\prime}, \tau_{M}\right)$ be an $R N$-space, $\left(Y, \Lambda, \tau_{M}\right)$ be a complete $R N$-space and $f: X \rightarrow Y$ be a mapping with $f(0)=0$ for which there is $\varphi:(X \backslash\{0\})^{3} \rightarrow Z$ such that

$$
\Lambda_{D f(x, y, z)}(t) \geq \Lambda_{\varphi(x, y, z)}^{\prime}(t)
$$

for all $x, y, z \in X \backslash\{0\}$ and $t>0$. If for all $x, y, z \in X \backslash\{0\}$ and $t>0 \varphi$ satisfies one of the following conditions: (i) $\Lambda_{\alpha \varphi(x, y, z)}^{\prime}(t) \leq \Lambda_{\varphi(2 x, 2 y, 2 z)}^{\prime}(t)$ for some $0<\alpha<2$, (ii) $\Lambda_{\varphi(2 x, 2 y, 2 z)}^{\prime}(t) \leq \Lambda_{\alpha \varphi(x, y, z)}^{\prime}(t)$ for some $4<\alpha$ then there exists a unique quadratic-additive mapping $F: X \rightarrow Y$ such that

$$
\Lambda_{f(x)-F(x)}(t) \geq \begin{cases}M(x,(2-\alpha) t) & \text { if } \varphi \text { satisfies }(\mathrm{i}), \\ M(x,(\alpha-4) t) & \text { if } \varphi \text { satisfies }(\mathrm{ii})\end{cases}
$$

for all $x \in X$ and $t>0$, where

$$
M(x, t):=\tau_{M}\left\{\Lambda_{\varphi(x, x,-x)}^{\prime}(t), \Lambda_{\varphi(-x,-x, x)}^{\prime}(t)\right\} .
$$

Moreover if $\alpha<1$ and $\Lambda_{\varphi(x, y, z)}^{\prime}$ is continuous in $x, y, z$ under the condition (i), then $f$ is a quadratic-additive mapping.

Proof. We will prove the theorem in two cases, $\varphi$ satisfies the condition (i) or (ii).

Case 1. Assume that $\varphi$ satisfies the condition (i). Let $S$ be the set of all functions $g: X \rightarrow Y$ with $g(0)=0$ and introduce a generalized metric on $S$ by

$$
d(g, h)=\inf \left\{u \in \mathbb{R}^{+} \mid \Lambda_{g(x)-h(x)}(u t) \geq M(x, t) \text { for all } x \in X \backslash\{0\}\right\} .
$$

Consider the mapping $J: S \rightarrow S$ defined by

$$
J f(x):=\frac{f(2 x)-f(-2 x)}{4}+\frac{f(2 x)+f(-2 x)}{8}
$$

then we have

$$
J^{n} f(x)=\frac{1}{2}\left(4^{-n}\left(f\left(2^{n} x\right)+f\left(-2^{n} x\right)\right)+2^{-n}\left(f\left(2^{n} x\right)-f\left(-2^{n} x\right)\right)\right)
$$

for all $x \in X$. Let $f, g \in S$ and let $u \in[0, \infty]$ be an arbitrary constant with $d(g, f) \leq u$. From the definition of $d,(\mathrm{RN} 2)$, and (RN3), for the given $0<\alpha<2$ we have

$$
\begin{aligned}
\Lambda_{J g(x)-J f(x)}\left(\frac{\alpha u}{2} t\right) & =\Lambda_{\frac{3(g(2 x)-f(2 x))}{8}-\frac{g(-2 x)-f(-2 x)}{8}}\left(\frac{\alpha u}{2} t\right) \\
& \geq \tau_{M}\left\{\Lambda_{\frac{3(g(2 x)-f(2 x))}{8}}\left(\frac{3 \alpha u t}{8}\right), \Lambda_{\frac{g(-2 x)-f(-2 x)}{8}}\left(\frac{\alpha u t}{8}\right)\right\} \\
& \geq \tau_{M}\left\{\Lambda_{g(2 x)-f(2 x)}(\alpha u t), \Lambda_{g(-2 x)-f(-2 x)}(\alpha u t)\right\}
\end{aligned}
$$




$$
\begin{aligned}
& \geq \tau_{M}\left\{\Lambda_{\varphi(2 x, 2 x,-2 x)}^{\prime}(\alpha t), \Lambda_{\varphi(-2 x,-2 x, 2 x)}^{\prime}(\alpha t)\right\} \\
& \geq M(x, t)
\end{aligned}
$$

for all $x \in X \backslash\{0\}$, which implies that

$$
d(J f, J g) \leq \frac{\alpha}{2} d(f, g) .
$$

That is, $J$ is a strictly contractive self-mapping of $S$ with the Lipschitz constant $0<\frac{\alpha}{2}<1$. Moreover, by (3.1), we see that

$$
\begin{aligned}
\Lambda_{f(x)-J f(x)}\left(\frac{t}{2}\right) & =\Lambda_{\frac{3 D f(x, x,-x)}{8}-\frac{D f(-x,-x, x)}{8}}\left(\frac{t}{2}\right) \\
& \geq \tau_{M}\left\{\Lambda_{\frac{3 D f(x, x,-x)}{8}}\left(\frac{3 t}{8}\right), \Lambda_{\frac{D f(-x,-x, x)}{8}}\left(\frac{t}{8}\right)\right\} \\
& \geq \tau_{M}\left\{\Lambda_{D f(x, x,-x)}(t), \Lambda_{D f(-x,-x, x)}(t)\right\} \\
& \geq \tau_{M}\left\{\Lambda_{\varphi(x, x,-x)}^{\prime}(t), \Lambda_{\varphi(-x,-x, x)}^{\prime}(t)\right\}
\end{aligned}
$$

for all $x \in X \backslash\{0\}$. It means that $d(f, J f) \leq \frac{1}{2}<\infty$ by the definition of $d$. Therefore according to Theorem 3.1, the sequence $\left\{J^{n} f\right\}$ converges to the unique fixed point $F: X \rightarrow Y$ of $J$ in the set $T=\{g \in S \mid d(f, g)<\infty\}$, which is represented by

$$
F(x):=\lim _{n \rightarrow \infty}\left(\frac{f\left(2^{n} x\right)+f\left(-2^{n} x\right)}{2 \cdot 4^{n}}+\frac{f\left(2^{n} x\right)-f\left(-2^{n} x\right)}{2^{n+1}}\right)
$$

for all $x \in V$. Since

$$
d(f, F) \leq \frac{1}{1-\frac{\alpha}{2}} d(f, J f) \leq \frac{1}{2-\alpha}
$$

the inequality (3.2) holds. Next we will show that $F$ is a quadratic-additive mapping. Let $x, y, z \in X$. Then by (RN3) we have

$$
\begin{aligned}
\Lambda_{D F(x, y, z)}(t) \geq \tau_{M}\{ & \Lambda_{\left(F-J^{n} f\right)(x+y+z)}\left(\frac{t}{14}\right), \Lambda_{\left(F-J^{n} f\right)(x)}\left(\frac{t}{14}\right), \\
& \Lambda_{\left(F-J^{n} f\right)(y)}\left(\frac{t}{14}\right), \Lambda_{\left(F-J^{n} f\right)(z)}\left(\frac{t}{14}\right), \Lambda_{\left(F-J^{n} f\right)(x+y)}\left(\frac{t}{14}\right), \\
& \left.\Lambda_{\left(F-J^{n} f\right)(x+z)}\left(\frac{t}{14}\right), \Lambda_{\left(F-J^{n} f\right)(y+z)}\left(\frac{t}{14}\right), \Lambda_{D J^{n} f(x, y, z)}\left(\frac{t}{2}\right)\right\}
\end{aligned}
$$

for all $x, y, z \in X \backslash\{0\}$ and $n \in \mathbb{N}$. The first seven terms on the right hand side of the above ineuality tend to 1 as $n \rightarrow \infty$ by the definition of $F$. Now consider that

$$
\Lambda_{D J^{n} f(x, y, z)}\left(\frac{t}{2}\right) \geq \tau_{M}\left\{\Lambda_{\frac{D f\left(2^{n} x, 2^{n} y, 2^{n} z\right)}{2 \cdot 4^{n}}}\left(\frac{t}{8}\right), \Lambda_{\frac{D f\left(-2^{n} x,-2^{n} y,-2^{n} z\right)}{2 \cdot 4^{n}}}\left(\frac{t}{8}\right),\right.
$$




$$
\begin{gathered}
\left.\Lambda_{\frac{D f\left(2^{n} x, 2^{n} y, 2^{n} z\right)}{2 \cdot 2^{n}}}\left(\frac{t}{8}\right), \Lambda_{\frac{D f\left(-2^{n} x,-2^{n} y,-2^{n} z\right)}{2^{n} 2^{n}}}\left(\frac{t}{8}\right)\right\} \\
\geq \tau_{M}\left\{\Lambda_{D f\left(2^{n} x, 2^{n} y, 2^{n} z\right)}\left(\frac{4^{n} t}{4}\right), \Lambda_{D f\left(-2^{n} x,-2^{n} y,-2^{n} z\right)}\left(\frac{4^{n} t}{4}\right),\right. \\
\left.\Lambda_{D f\left(2^{n} x, 2^{n} y, 2^{n} z\right)}\left(\frac{2^{n} t}{4}\right), \Lambda_{D f\left(-2^{n} x,-2^{n} y,-2^{n} z\right)}\left(\frac{2^{n} t}{4}\right)\right\} \\
\geq \tau_{M}\left\{\Lambda_{\varphi(x, y, z)}^{\prime}\left(\frac{4^{n} t}{4 \alpha^{n}}\right), \Lambda_{\varphi(-x,-y,-z)}^{\prime}\left(\frac{4^{n} t}{4 \alpha^{n}}\right),\right. \\
\left.\Lambda_{\varphi(x, y, z)}^{\prime}\left(\frac{2^{n} t}{4 \alpha^{n}}\right), \Lambda_{\varphi(-x,-y,-z)}^{\prime}\left(\frac{2^{n} t}{4 \alpha^{n}}\right)\right\}
\end{gathered}
$$

which tends to 1 as $n \rightarrow \infty$ by (RN3) and $\frac{2}{\alpha}>1$ for all $x, y, z \in X \backslash\{0\}$. Therefore it follows from (3.3) that

$$
\Lambda_{D F(x, y, z)}(t)=1
$$

for each $x, y, z \in X \backslash\{0\}$ and $t>0$. By (RN1) and Lemma 3.2, this means that $D F(x, y, z)=0$ for all $x, y, z \in X$. Assume that $\alpha<1$ and $\Lambda_{\varphi(x, y, z)}^{\prime}$ is continuous in $x, y, z$. If $m, a_{1}, b_{1}, a_{2}, b_{2}, a_{3}, b_{3}$ are any fixed integers with $a_{1}, a_{2}, a_{3} \neq 0$, then we have

$$
\begin{aligned}
\lim _{n \rightarrow \infty} \Lambda_{\varphi\left(\left(2^{n} a_{1}+b_{1}\right) x,\left(2^{n} a_{2}+b_{2}\right) y,\left(2^{n} a_{3}+b_{3}\right) z\right)}^{\prime}(t) \\
\quad \geq \lim _{n \rightarrow \infty} \Lambda_{\varphi\left(\left(a_{1}+\frac{b_{1}}{2^{n}}\right) x,\left(a_{2}+\frac{b_{2}}{2^{n}}\right) y,\left(a_{3}+\frac{b_{3}}{2^{n}}\right) z\right)}^{\prime}\left(\frac{t}{\alpha^{n}}\right) \\
\geq \lim _{n \rightarrow \infty} \Lambda_{\varphi\left(\left(a_{1}+\frac{b_{1}}{2^{n}}\right) x,\left(a_{2}+\frac{b_{2}}{2^{n}}\right) y,\left(a_{3}+\frac{b_{3}}{2^{n}}\right) z\right)}^{(m t)} \\
\quad=\Lambda_{\varphi\left(a_{1} x, a_{2} y, a_{3} z\right)}^{\prime}(m t)
\end{aligned}
$$

for all $x, y, z \in X \backslash\{0\}$ and $t>0$. Since $m$ is arbitrary, we have

$$
\lim _{n \rightarrow \infty} \Lambda_{\varphi\left(\left(2^{n} a_{1}+b_{1}\right) x,\left(2^{n} a_{2}+b_{2}\right) y,\left(2^{n} a_{3}+b_{3}\right) z\right)}^{\prime}(t) \geq \lim _{m \rightarrow \infty} \Lambda_{\varphi\left(a_{1} x, a_{2} y, a_{3} z\right)}^{\prime}(m t)=1
$$

for all $x, y, z \in X \backslash\{0\}$ and $t>0$. From these, we get the inequality

$$
\begin{gathered}
\Lambda_{F(x)-f(x)}(6 t) \geq \lim _{n \rightarrow \infty} \tau_{M}\left\{\Lambda_{(D f-D F)\left(\left(2^{n}+1\right) x, 2^{n} x,-2^{n} x\right)}(t),\right. \\
\Lambda_{2(F-f)\left(\left(2^{n}+1\right) x\right)}(2 t), \Lambda_{(f-F)\left(\left(2^{n+1}+1\right) x\right)}(t), \\
\left.\Lambda_{(F-f)\left(2^{n} x\right)}(t), \Lambda_{(F-f)\left(-2^{n} x\right)}(t)\right\} \\
\geq \lim _{n \rightarrow \infty} \tau_{M}\left\{\Lambda_{\varphi\left(\left(2^{n}+1\right) x, 2^{n} x,-2^{n} x\right)}^{\prime}(t),\right.
\end{gathered}
$$




$$
\begin{aligned}
& M\left(\left(2^{n}+1\right) x,(2-\alpha) t\right), M\left(\left(2^{n+1}+1\right) x,(2-\alpha) t\right), \\
& \left.M\left(2^{n} x,(2-\alpha) t\right), M\left(-2^{n} x,(2-\alpha) t\right)\right\}=1
\end{aligned}
$$

for all $x \in X \backslash\{0\}$. From the above inequality and the fact $f(0)=0=F(0)$, we obtain $f \equiv F$. This completes the proof of this theorem.

Case 2. We take $\alpha>4$ and let $\varphi$ satisfy the condition (ii). Let the set $(S, d)$ be as in the proof of Case 1. Now we consider the mapping $J: S \rightarrow S$ defined by

$$
J g(x):=g\left(\frac{x}{2}\right)-g\left(-\frac{x}{2}\right)+2\left(g\left(\frac{x}{2}\right)+g\left(-\frac{x}{2}\right)\right)
$$

for all $g \in S$ and $x \in V$. Notice that

$$
J^{n} g(x)=2^{n-1}\left(g\left(\frac{x}{2^{n}}\right)-g\left(-\frac{x}{2^{n}}\right)\right)+\frac{4^{n}}{2}\left(g\left(\frac{x}{2^{n}}\right)+g\left(-\frac{x}{2^{n}}\right)\right)
$$

for all $x \in X$. Let $f, g \in S$ and let $u \in[0, \infty]$ be an arbitrary constant with $d(g, f) \leq u$. From the definition of $d,(\mathrm{RN} 2)$, and (RN3), we have

$$
\begin{aligned}
\Lambda_{J g(x)-J f(x)}\left(\frac{4 u}{\alpha} t\right) & =\Lambda_{3\left(g\left(\frac{x}{2}\right)-f\left(\frac{x}{2}\right)\right)+g\left(-\frac{x}{2}\right)-f\left(-\frac{x}{2}\right)}\left(\frac{4 u}{\alpha} t\right) \\
& \geq \tau_{M}\left\{\Lambda_{3\left(g\left(\frac{x}{2}\right)-f\left(\frac{x}{2}\right)\right)}\left(\frac{3 u}{\alpha} t\right), \Lambda_{g\left(-\frac{x}{2}\right)-f\left(-\frac{x}{2}\right)}\left(\frac{u}{\alpha} t\right)\right\} \\
& \geq \tau_{M}\left\{\Lambda_{g\left(\frac{x}{2}\right)-f\left(\frac{x}{2}\right)}\left(\frac{u}{\alpha} t\right), \Lambda_{g\left(-\frac{x}{2}\right)-f\left(-\frac{x}{2}\right)}\left(\frac{u}{\alpha} t\right)\right\} \\
& \geq \tau_{M}\left\{\Lambda_{\varphi\left(\frac{x}{2}, \frac{x}{2},-\frac{x}{2}\right)}^{\prime}\left(\frac{t}{\alpha}\right), \Lambda_{\varphi\left(-\frac{x}{2},-\frac{x}{2}, \frac{x}{2}\right)}^{\prime}\left(\frac{t}{\alpha}\right)\right\} \\
& \geq M(x, t)
\end{aligned}
$$

for all $x \in X \backslash\{0\}$, which implies that

$$
d(J f, J g) \leq \frac{4}{\alpha} d(f, g) .
$$

That is, $J$ is a strictly contractive self-mapping of $S$ with the Lipschitz constant $0<\frac{4}{\alpha}<1$. Moreover, by (3.1), we see that

$$
\begin{aligned}
\Lambda_{f(x)-J f(x)}\left(\frac{t}{\alpha}\right) & =\Lambda_{-D f\left(\frac{x}{2}, \frac{x}{2},-\frac{x}{2}\right)}\left(\frac{t}{\alpha}\right) \\
& \geq \Lambda_{\varphi\left(\frac{x}{2}, \frac{x}{2},-\frac{x}{2}\right)}^{\prime}\left(\frac{t}{\alpha}\right) \\
& \geq \Lambda_{\varphi(x, x,-x)}^{\prime}(t)
\end{aligned}
$$

for all $x \in X \backslash\{0\}$. It means that $d(f, J f) \leq \frac{1}{\alpha}<\infty$ by the definition of $d$. Therefore according to Theorem 3.1, the sequence $\left\{J^{n} f\right\}$ converges to the unique fixed point 
$F: X \rightarrow Y$ of $J$ in the set $T=\{g \in S \mid d(f, g)<\infty\}$, which is represented by

$$
F(x):=\lim _{n \rightarrow \infty}\left(2^{n-1}\left(f\left(\frac{x}{2^{n}}\right)-f\left(-\frac{x}{2^{n}}\right)\right)+\frac{4^{n}}{2}\left(f\left(\frac{x}{2^{n}}\right)+f\left(-\frac{x}{2^{n}}\right)\right)\right)
$$

for all $x \in X$. Since

$$
d(f, F) \leq \frac{1}{1-\frac{4}{\alpha}} d(f, J f) \leq \frac{1}{\alpha-4}
$$

the inequality (3.2) holds. Next we will show that $F$ is quadratic-additive. Let $x, y, z \in X$. Then by (RN3) we have the inequality (3.3) for all $x, y, z \in X \backslash\{0\}$ and $n \in \mathbb{N}$. The first seven terms on the right hand side of the inequality (3.3) tend to 1 as $n \rightarrow \infty$ by the definition of $F$. Now consider that

$$
\begin{aligned}
& \Lambda_{D J^{n} f(x, y, z)}\left(\frac{t}{2}\right) \geq \tau_{M}\left\{\Lambda_{2^{2 n-1} D f\left(\frac{x}{2^{n}}, \frac{y}{2^{n}}, \frac{z}{2^{n}}\right)}\left(\frac{t}{8}\right), \Lambda_{2^{2 n-1} D f\left(\frac{-x}{2^{n}}, \frac{-y}{2^{n}}, \frac{-z}{2^{n}}\right)}\left(\frac{t}{8}\right),\right. \\
& \left.\Lambda_{2^{n-1} D f\left(\frac{x}{2^{n}}, \frac{y}{2^{n}}, \frac{z}{2^{n}}\right)}\left(\frac{t}{8}\right), \Lambda_{-2^{n-1} D f\left(\frac{-x}{2^{n}}, \frac{-y}{2^{n}}, \frac{-z}{2^{n}}\right)}\left(\frac{t}{8}\right)\right\} \\
& \geq \tau_{M}\left\{\Lambda_{\varphi(x, y, z)}^{\prime}\left(\frac{\alpha^{n} t}{4^{n+1}}\right), \Lambda_{\varphi(-x,-y,-z)}^{\prime}\left(\frac{\alpha^{n} t}{4^{n+1}}\right),\right. \\
& \left.\Lambda_{\varphi(x, y, z)}^{\prime}\left(\frac{\alpha^{n} t}{2^{n+2}}\right), \Lambda_{\varphi(-x,-y,-z)}^{\prime}\left(\frac{\alpha^{n} t}{2^{n+2}}\right)\right\}
\end{aligned}
$$

which tends to 1 as $n \rightarrow \infty$ by (RN3) for all $x, y, z \in X \backslash\{0\}$. Therefore it follows from (3.3) that

$$
\Lambda_{D F(x, y, z)}(t)=1
$$

for each $x, y, z \in X \backslash\{0\}$ and $t>0$. By (RN1) and Lemma 3.1, this means that $D F(x, y, z)=0$ for all $x, y, z \in X$. It completes the proof of Theorem 3.3.

Now we will establish the stability for the functional equations (1.3) in random normed spaces.

Theorem 3.4. Let $X,\left(Z, \Lambda^{\prime}, \tau_{M}\right),\left(Y, \Lambda, \tau_{M}\right), \varphi$ and $M(x, t)$ be as in Theorem 3.3. If $f: X \rightarrow Y$ is a mapping such that

$$
\Lambda_{D^{\prime} f(x, y, z)}(t) \geq \Lambda_{\varphi(x, y, z)}^{\prime}(t)
$$

for all $x, y, z \in X \backslash\{0\}$ and $t>0$, then there exists a unique general quadratic mapping $F: X \rightarrow Y$ satisfying $F(0)=f(0)$ and (3.2) for all $x \in X \backslash\{0\}$ and $t>0$.

Proof. Let $\tilde{f}=f-f(0)$. Then by (3.4) we have

$$
\Lambda_{D \tilde{f}(x, y, z)}(t)=\Lambda_{D^{\prime} f(x, y, z)}(t) \geq \Lambda_{\varphi(x, y, z)}^{\prime}(t)
$$

for all $x, y, z \in X \backslash\{0\}$ and $t>0$ with $\tilde{f}(0)=0$. By Theorem 3.3, there exists a unique mapping $\tilde{F}: X \rightarrow Y$ satisfying (3.2) for $\tilde{f}$ and $D \tilde{F}(x, y, z)=0$. Put 
$F=\tilde{F}+f(0)$, then we easily show that $D^{\prime} F(x, y, z)=0$ and $F$ satisfying (3.2) for $f$.

Now we have a generalized Hyers-Ulam stability of the quadratic-additive functional equation (1.2) in the framework of normed spaces. Let $\Lambda_{x}(t)=\frac{t}{t+\|x\|}$. Then $\left(X, \Lambda, \tau_{M}\right)$ is an induced random normed space, which leads us to get the following result.

Corollary 3.5. Let $X$ be a linear space and $Y$ a complete normed-space. And let $f: X \rightarrow Y$ be a mapping with $f(0)=0$ for which there is $\varphi:(X \backslash\{0\})^{3} \rightarrow[0, \infty)$ such that

$$
\|D f(x, y, z)\| \leq \varphi(x, y, z)
$$

for all $x, y, z \in X \backslash\{0\}$. If, for all $x, y, z \in X \backslash\{0\}, \varphi$ satisfies one of the following conditions:

(i) $\alpha \varphi(x, y, z) \geq \varphi(2 x, 2 y, 2 z)$ for some $0<\alpha<2$,

(ii) $\varphi(2 x, 2 y, 2 z) \geq \alpha \varphi(x, y, z)$ for some $4<\alpha$

then there exists a unique quadratic-additive mapping $F: X \rightarrow Y$ such that

$$
\|f(x)-F(x)\| \leq \begin{cases}\frac{\Phi(x)}{2-\alpha} & \text { if } \varphi \text { satisfies (i), } \\ \frac{\Phi(x)}{\alpha-4} & \text { if } \varphi \text { satisfies (ii) }\end{cases}
$$

for all $x \in X \backslash\{0\}$, where $\Phi(x)$ is defined by

$$
\Phi(x)=\max (\varphi(x, x,-x), \varphi(-x,-x, x)) .
$$

Moreover, if $0<\alpha<1$ and $\varphi$ is continuous under the condition (i), then $f$ is a quadratic-additive mapping.

Now we have a Hyers-Ulam-Rassias stability of the quadratic-additive functional equation (1.2).

Corollary 3.6. Let $X$ be a normed space, $p, q, r \in[0,1) \cup(2, \infty)$ and $Y$ a complete normed-space. If $f: X \rightarrow Y$ is a mapping such that

$$
\|D f(x, y, z)\| \leq\|x\|^{p}+\|y\|^{q}+\|z\|^{r}
$$

for all $x, y, z \in X \backslash\{0\}$ with $f(0)=0$, then there exists a unique quadratic-additive mapping $F: X \rightarrow Y$ such that

$$
\|f(x)-F(x)\| \leq \begin{cases}\frac{\|x\|^{p}+\|x\|^{q}+\|x\|^{r}}{2-2^{\max \{p, q, r\}}} & \text { if } 0 \leq p, q, r<1, \\ \frac{\|x\|^{p}+\|x\|^{q}+\|x\|^{r}}{2^{\min \{p, q, r\}}-4} & \text { if } 2<p, q, r\end{cases}
$$

for all $x \in X \backslash\{0\}$. 
Proof. It follows from Corollary 3.5 by putting $\varphi(x, y, z)=\|x\|^{p}+\|y\|^{q}+\|z\|^{r}$ with $\alpha=2^{\max \{p, q, r\}}<2$ if $0 \leq p, q, r<1$ and $\alpha=2^{\min \{p, q, r\}}>4$ if $p, q, r>2$.

Corollary 3.7. Let $X$ be a normed space, $p, q, r<0$ and $Y$ a complete normedspace. If $f: X \rightarrow Y$ is a mapping satisfying (3.6) for all $x, y, z \in X \backslash\{0\}$, then $f$ is itself a quadratic-additive mapping.

Proof. By choosing a fixed $x \in X \backslash\{0\}$, we have

$$
\begin{aligned}
\|2 f(0)\| & =\lim _{n \rightarrow \infty}\left\|D f\left(2^{n+1} x,-2^{n} x,-2^{n} x\right)+D f\left(-2^{n+1} x, 2^{n} x, 2^{n} x\right)\right\| \\
& \leq \lim _{n \rightarrow \infty} 2\left(\left\|2^{n+1} x\right\|^{p}+\left\|2^{n} x\right\|^{q}+\left\|2^{n} x\right\|^{r}\right) \\
& \leq \lim _{n \rightarrow \infty} 2^{n p}\left(2\|2 x\|^{p}+2\|x\|^{q}+2\|x\|^{r}\right)=0 .
\end{aligned}
$$

It follows from Corollary 3.5 by putting $\varphi(x, y, z)=\|x\|^{p}+\|y\|^{q}+\|z\|^{r}$ with $\alpha=$ $2^{\max \{p, q, r\}}<1$.

We have a general Hyers-Ulam stability results of the general quadratic functional equation (1.3) as a corollary of Theorem 3.4.

Corollary 3.8. Let $X, Y, \varphi$ and $\Phi(x)$ be as in Theorem 3.3. If $f: X \rightarrow Y$ is a mapping such that

$$
\left\|D^{\prime} f(x, y, z)\right\| \leq \varphi(x, y, z)
$$

for all $x, y, z \in X \backslash\{0\}$, then there exists a unique general quadratic mapping $F$ : $X \rightarrow Y$ such that $F(0)=f(0)$ and (3.5) holds.

Now we have Hyers-Ulam-Rassias stability of the general quadratic functional equation (1.3).

Corollary 3.9. Let $X$ be a normed space, $p, q, r \in[0,1) \cup(2, \infty)$, and $Y$ a complete normed-space. If $f: X \rightarrow Y$ is a mapping such that

$$
\left\|D^{\prime} f(x, y, z)\right\| \leq\|x\|^{p}+\|y\|^{q}+\|z\|^{r}
$$

for all $x, y, z \in X \backslash\{0\}$, then there exists a unique general quadratic mapping $F$ : $X \rightarrow Y$ such that $F(0)=f(0)$ and (3.7) holds.

Corollary 3.10. Let $X$ be a normed space, $p, q, r<0$ and $Y$ a complete normedspace. If $f: X \rightarrow Y$ is a mapping satisfying (3.8) for all $x, y, z \in X \backslash\{0\}$, then $f$ is itself a general quadratic mapping. 


\section{REFERENCES}

1. T. Aoki: On the stability of the linear transformation in Banach spaces. J. Math. Soc. Japan 2 (1950), 64-66.

2. P.W. Cholewa: Remarks on the stability of functional equations. Aequationes Math. $\mathbf{2 7}$ (1984), 76-86.

3. Z. Gajda: On stability of additive mappings. Internat. J. Math. Math. Sci. 14 (1991), 431-434.

4. P. Găvruta: A generalization of the Hyers-Ulam-Rassias stability of approximately additive mappings. J. Math. Anal. Appl. 184 (1994), 431-436.

5. D. H. Hyers: On the stability of the linear functional equation. Proc. Natl. Acad. Sci. 27 (1941), 222-224.

6. S.-S. Jin \& Y.-H. Lee: A fixed point approach to the stability of the mixed type functional equation. to appear

7. S.-M. Jung: On the Hyers-Ulam stability of the functional equations that have the quadratic property. J. Math. Anal. Appl. 222 (1998), 126-137.

8. H.-M. Kim: On the stability problem for a mixed type of quartic and quadratic functional equation. J. Math. Anal. Appl. 324 (2006), 358-372.

9. Y.-H. Lee: On the stability of the monomial functional equation. Bull. Korean Math. Soc. 45 (2008), 397-403.

10. Y.-H. Lee \& K.-W. Jun: A generalization of the Hyers-Ulam-Rassias stability of Jensen's equation. J. Math. Anal. Appl. 238 (1999), 305-315.

11. _ : A generalization of the Hyers-Ulam-Rassias stability of Pexider equation. $J$. Math. Anal. Appl. 246 (2000), 627-638.

12. _ : On the stability of approximately additive mappings. Proc. Amer. Math. Soc. 128 (2000), 1361-1369.

13. B. Margolis \& J.B. Diaz: A fixed point theorem of the alternative for contractions on a generalized complete metric space. Bull. Amer. Math. Soc. 74 (1968), 305-309.

14. D. Mihet \& V. Radu: On the stability of the additive Cauchy functional equation in random normed spaces. J. Math. Anal. Appl. 343 (2008), no. 1, 567-572.

15. V. Radu: The fixed point alternative and the stability of functional equations. Sem. Fixed Point Theory 4 (2003), no. 1, 91-96.

16. Th. M. Rassias: On the stability of the linear mapping in Banach spaces. Proc. Amer. Math. Soc. 72 (1978), 297-300.

17. I.A. Rus: Principles and applications of fixed point theory. Ed. Dacia, Cluj-Napoca. 1979(in Romanian).

18. B. Schweizer \& A. Sklar: Probabilistic metric spaces. Noth-Holland Series in Probability and Applied Mathematics, North-Holland, New York, NY, USA (1983). 
19. A.N. S̆erstnev: On the motion of a random normed space. Dokl. Akad. Nauk SSSR 149 (1963), 280-283.

20. S.M. Ulam: A collection of mathematical problems. Interscience, New York, 1968, p. 63.

${ }^{a}$ Department of Mathematics Education, Gonguu National University of Education, GONGJU 314-711, KOREA

Email address: ssjin@gjue.ac.kr

${ }^{b}$ Department of Mathematics Education, Gonguu National University of Education, GONGJU 314-711, KOREA

Email address: yanghi2@hanmail.net 